\title{
Embedded vortices
}

\author{
Nathan F. Lepora* \\ Department of Applied Mathematics and Theoretical Physics, University of Cambridge, Silver Street, Cambridge CB3 $9 E W$, \\ United Kingdom \\ Anne-Christine Davis ${ }^{\dagger}$ \\ Department of Applied Mathematics and Theoretical Physics, University of Cambridge, Silver Street, Cambridge CB3 $9 E W$, \\ United Kingdom \\ and Theory Division, CERN, Geneva 23, CH-1211, Switzerland
}

(Received 14 July 1995; revised manuscript received 4 April 1997; published 23 November 1998)

\begin{abstract}
We present a discussion of embedded vortices in general Yang-Mills theories. The origin of a family structure of solutions is shown to be group theoretic in nature and a procedure for its determination is developed. Vortex stability can be quantified into three types: Abelian topological stability, non-Abelian topological stability, and dynamical stability; we relate these to the family structure of vortices, in particular discussing how Abelian topological and dynamical stability are related. The formalism also generally encompasses embedded domain walls and embedded monopoles also. [S0556-2821(98)01018-2]
\end{abstract}

PACS number(s): 11.27. $+\mathrm{d}$

\section{INTRODUCTION}

In this paper we discuss how one determines the embedded vortex structure of a general Yang-Mills theory. The concept of an embedded defect, in a proper mathematical context, was introduced by Barriola, Vachaspati, and Bucher [1]; they consider embedded defects of the general form

$$
\begin{aligned}
& \text { (radial profile function) } \\
& \quad \times(\text { angular exponential of Lie subalgebra }) \text {. }
\end{aligned}
$$

The usual topological defects therefore fit into this class. However, one should also be aware that there are other defect solutions not of this form. We aim to discuss the spectrum and properties of vortex solutions of the embedded defect form above.

One should note that many examples of the formalism contained within this paper are given in a companion paper, which also contains the usual historical perspective [2].

\section{Prenote: Restriction to formalism}

Because of extra complications for a variety of symmetry breaking schemes we shall make the following restriction on theories considered in this paper: For symmetry breaking schemes of the form $G \rightarrow H$, write $\mathcal{G}=\mathcal{H} \oplus \mathcal{M}$ and split $\mathcal{M}$ into irreducible parts under $\operatorname{Ad}(H): \mathcal{M}=\mathcal{M}_{1} \oplus \cdots \oplus \mathcal{M}_{n}$.

Then we confine our discussion to those $\mathcal{M}_{i}$ 's such that

$$
\operatorname{rank}\left(\mathcal{M}_{i}\right)=1
$$

where the rank of $\mathcal{M}_{i}$ is defined to be the dimension of the maximal Abelian subalgebra within $\mathcal{M}_{i}$.

\footnotetext{
*Email address: N.F.Lepora@amtp.cam.ac.uk

†Email address: A.C.Davis@amtp.cam.ac.uk
}

The terminology in the above is explained within the paper. However, we feel it should be stated here so that one is aware of this restriction from the start.

\section{EMBEDDED VORTICES}

In order to provide an understanding of the nature and stability of embedded defects it is necessary to have a framework for describing them. This has been provided by Barriola, Vachaspati, and Bucher [1]. For work in this paper it is necessary to use the underlying group theory behind the theory of embedded defects. Thus, in this section we indicate how group theory connects with the formalism introduced in [1].

Consider a Yang-Mills field theory with gauge symmetry group $G$ that breaks to a smaller group $H$ via condensation of a scalar field $\Phi$ taking values in a vector space $\mathcal{V}$. One describes the action of $G$ upon $\mathcal{V}$ by the representation, $D$ of $G$. The corresponding Lie algebra, $\mathcal{G}$, acts upon $\mathcal{V}$ by the derived representation $d$, related to $D$ by $D\left(e^{X}\right)=e^{d(X)}$.

Such a field theory is specified by a Lagrangian describing the interaction of a gauge field $A^{\mu} \in \mathcal{G}$ with the scalar field $\Phi$ :

$$
\begin{aligned}
\mathcal{L}\left[\Phi, A^{\mu}\right]= & -\frac{1}{4}\left\langle F_{\mu \nu}, F^{\mu \nu}\right\rangle \\
& +\frac{1}{2}\left\langle\left(\partial_{\mu}+d\left(A_{\mu}\right)\right) \Phi\left(\partial^{\mu}+d\left(A^{\mu}\right)\right) \Phi\right\rangle-V[\Phi],
\end{aligned}
$$

with

$$
F_{\mu \nu}=\partial_{\mu} A_{\nu}-\partial_{\nu} A_{\mu}+\left[A_{\mu}, A_{\nu}\right],
$$

and $V$ the scalar potential. To give $\mathcal{L}$ the necessary symmetry properties the inner products $\langle.,$.$\rangle are constrained to be invari-$ ant under the action of $G$. Note that we are using the same symbol to denote both inner products; we hope it should be clear from the context which one we are using.

The inner product upon $\mathcal{V}$ is defined to be of the real form 


$$
\langle\Phi, \Psi\rangle=\operatorname{Re}\left(\Phi^{\dagger} \Psi\right)
$$

for invariance of the scalar field kinetic term under the group $G l(\mathcal{V})$.

The inner product upon $\mathcal{G}$ is defined to

$$
\langle., .\rangle=\frac{1}{q_{1}^{2}}\{., .\}_{1}+\cdots+\frac{1}{q_{n}^{2}}\{., .\}_{n},
$$

with $\{.,\}_{i}$ the inner product $\{X, Y\}=-p \operatorname{Re}\left(\operatorname{Tr}\left(X^{\dagger} Y\right)\right)$, restricted to $\mathcal{G}_{i}$, one of the mutually commuting subalgebras of $\mathcal{G}=\mathcal{G}_{1} \oplus \cdots \oplus \mathcal{G}_{n}$. The $n$ real scales $\left\{q_{i}\right\}$ are related to the gauge coupling constants of the theory. This inner product (4) is the most general $\operatorname{Ad}(G)$-invariant inner product, where Ad is the adjoint representation $\operatorname{Ad}(g) X=g X g^{-1}$ for $X$ $\in \mathcal{G}$.

A minima of $V$, say $\Phi_{0} \in \mathcal{V}$, is arbitrary because of the degeneracy of choice given by the vacuum manifold $M$ $=D(G) \Phi_{0}$. The vacuum manifold $M \cong G / H$, with $H$ the residual symmetry group determined by $\Phi_{0}$ to be $H=\{g$ $\left.\in G: D(g) \Phi_{0}=\Phi_{0}\right\}$. Then the inner product (4) defines the decomposition

$$
\mathcal{G}=\mathcal{H} \oplus \mathcal{M}
$$

with $\mathcal{H}$ the Lie algebra of $H$.

To construct an embedded defect solution on such a field theory one chooses a subgroup $G_{\mathrm{emb}} \subset G$ such that the corresponding homotopy group $\pi_{k}\left(G_{\mathrm{emb}} / H_{\mathrm{emb}}\right)$ is nontrivial, where $H_{\mathrm{emb}}=G_{\mathrm{emb}} \cap H$. The crucial idea is to find a smaller theory on which a defect is topological and then extend this solution back to the full theory. This gives rise to the concept of an embedded subtheory: a pair $\left(\mathcal{V}_{\mathrm{emb}}, G_{\mathrm{emb}}\right) \subset \mathcal{V} \times G$, with $\mathcal{V}_{\text {emb }}$ a minimal nontrivial vector subspace of $\mathcal{V}$ invariant un$\operatorname{der} D\left(G_{\mathrm{emb}}\right)$.

From the inner products (3), (4) we identify $\mathcal{V}_{\mathrm{emb}}^{\perp}$ and $\mathcal{G}_{\mathrm{emb}}^{\perp}$ as the corresponding orthogonal subspaces to $\mathcal{V}_{\text {emb }}$ and $\mathcal{G}_{\text {emb }}$ within $\mathcal{V}$ and $\mathcal{G}$, respectively.

The main result from [1] is that a defect topological in the embedded subspace

$$
\begin{array}{r}
\Phi(x)=\Phi_{\mathrm{emb}}(x) \in \mathcal{V}_{\mathrm{emb}}, \\
A^{\mu}(x)=A_{\mathrm{emb}}^{\mu}(x) \in \mathcal{G}_{\mathrm{emb}},
\end{array}
$$

remains a solution of the full theory provided the following conditions hold.

(1) The scalar potential satisfies

$$
\begin{aligned}
\frac{\partial V}{\partial \Psi}[\phi]:=\left\langle\Psi, \frac{\partial V}{\partial \Phi}[\phi]\right\rangle=0, & \\
& \text { for } \Psi \in \mathcal{V}_{\mathrm{emb}}^{\perp}, \phi \in \mathcal{V}_{\mathrm{emb}}, \Phi \in \mathcal{V} .
\end{aligned}
$$

(2) The representation satisfies

$$
d\left(X^{\perp}\right) \Phi \in \mathcal{V}_{\mathrm{emb}}^{\perp}, \quad \text { for } \Phi \in \mathcal{V}_{\mathrm{emb}} \quad \text { and } X^{\perp} \in \mathcal{G}_{\mathrm{emb}}^{\perp}
$$

These two conditions are provided by a direct substitution of Eqs. (6a), (6b) into the field equations, requiring that "embedded fields do not induce non-embedded currents" [1].

We now specialize to vortex solutions. In doing so we will shift the emphasis away from defining the embedded vortex by the pair $\left(\mathcal{V}_{\mathrm{emb}}, G_{\mathrm{emb}}\right)$; instead we consider the equivalent pair $\left(\Phi_{0}, X\right) \in \mathcal{V}_{\mathrm{emb}} \times \mathcal{G}_{\mathrm{emb}}$. form

An embedded vortex defined by the pair $\left(\Phi_{0}, X\right)$ is of the

$$
\begin{aligned}
& \Phi(r, \theta)=f_{\mathrm{NO}}(X ; r) \exp (d(X) \theta) \Phi_{0}, \\
& A(r, \theta)=\frac{g_{\mathrm{NO}}(X ; r)}{r} X \underline{\hat{\theta}} .
\end{aligned}
$$

The two profile functions $f_{\mathrm{NO}}$ and $g_{\mathrm{NO}}$ depend upon the generator of the embedded defect, and we discuss how so in the Appendix. One also has the single valuedness constraint that

$$
\exp (2 \pi d(X)) \Phi_{0}=\Phi_{0}
$$

The above vortex (9a), (9b) represents the minimum energy configuration of this form for $X \in \mathcal{M}$, since if we were to add any component in $\mathcal{H}$ the effect would be to leave the scalar field unchanged but to add an extra term to the gaugefield magnetic energy. Thus we restrict $\left(\Phi_{0}, X\right) \in \mathcal{V} \times \mathcal{M}$.

It is conceptually important to relate the above description of embedded vortices to the formalism of embedded defects. There is a natural correspondence between defining a vortex from the pair $\left(\mathcal{V}_{\mathrm{emb}}, \mathcal{G}_{\mathrm{emb}}\right)$ to the pair $\left(\Phi_{0}, X\right)$ provided by the following relations:

$$
\begin{aligned}
& \mathcal{G}_{\mathrm{emb}}=\mathbf{R} X, \\
& G_{\mathrm{emb}}=\exp (\mathbf{R} X), \\
& \mathcal{V}_{\mathrm{emb}}=\mathbf{R} D\left(G_{\mathrm{emb}}\right) \Phi_{0},
\end{aligned}
$$

with $\mathbf{R}$ the real number line.

Applying Eqs. (7), (8) for existence of embedded defects to the above description of embedded vortices we see that Eq. (7) is satisfied provided that a suitable potential is chosen; this condition is essentially a restriction on the form of the field theory. The condition (8) is more fundamental; we prove below that Eq. (8) is equivalent to the condition that $X$ be a vortex generator provided

$$
\left\langle d(X) \Phi_{0}, d\left(X^{\perp}\right) \Phi_{0}\right\rangle=0,
$$

for $\left\langle X^{\perp}, X\right\rangle=0$. This has the rather nice interpretation that only subspaces over which the derived representation is conformal are admissible to define embedded vortices. I.e., viewing $d(X) \Phi_{0}$ as a map between $\mathcal{G}$ and $\mathcal{V}$, Eq. (12) says that this map must preserve orthogonality with respect to Eq. (3) from the domains $\mathcal{G}_{\mathrm{emb}}$ and $\mathcal{G}_{\mathrm{emb}}^{\perp}$. 
We now prove the equivalence of conditions (8) and (12): writing $\mathcal{V}_{\mathrm{emb}}=\mathbf{R} \Phi \oplus \mathbf{R} d(X) \Phi$ we see that for $\Phi \in \mathcal{V}_{\mathrm{emb}}$ and $\left\langle X^{\perp}, X\right\rangle=0$,

$$
\begin{aligned}
d\left(X^{\perp}\right) \Phi \in \mathcal{V}_{\mathrm{emb}}^{\perp} & \Leftrightarrow\left(\mathcal{V}_{\mathrm{emb}}, d\left(X^{\perp}\right) \Phi\right)=0 \\
& \Leftrightarrow\left\langle d(X) \Phi, d\left(X^{\perp}\right) \Phi\right\rangle=0 \\
& \Leftrightarrow\left\langle d(X) \Phi_{0}, d\left(X^{\perp}\right) \Phi_{0}\right\rangle=0 .
\end{aligned}
$$

The second step by virtue of $\langle d(\mathcal{G}) \Phi, \Phi\rangle=0$, and the last by virtue of for general $\Phi \in \mathcal{V}_{\text {emb }}$ there exists a $g \in G_{\text {emb }}$ which rotates the above $\Phi$ to a constant times $\Phi_{0}$. This transformation leaves $\mathcal{G}_{\text {emb }}$ and $\mathcal{G}_{\text {emb }}^{\perp}$ invariant, because $G_{\text {emb }}$ is a group.

\section{FAMILIES OF EMBEDDED VORTICES}

We will now determine the family structure of embedded vortices in a general Yang-Mills theory. This essentially boils down to a description of group actions of $G$ upon the vacuum manifold. To describe this properly requires some rather technical work, and so, for pedagogical reasons, we shall assume some of the more technical points and cover a proper, more formal treatment in another paper [3].

In summary of the previous section: an embedded vortex is defined by the pair $\left(\Phi_{0}, X\right)$ to be

$$
\begin{aligned}
& \Phi(r, \theta)=f_{\mathrm{NO}}(X ; r) \exp (d(X) \theta) \Phi_{0}, \\
& \underline{A}(r, \theta)=\frac{g_{\mathrm{NO}}(X ; r)}{r} X \underline{\hat{\theta}},
\end{aligned}
$$

which is a solution provided

$$
\begin{aligned}
\frac{\partial V}{\partial \Psi}[\Phi] & =0, \quad \text { for } \Psi \in \mathcal{V}_{\mathrm{emb}}^{\perp}, \Phi \in \mathcal{V}_{\mathrm{emb}}, \\
\left\langle d(X) \Phi_{0}, d\left(X^{\perp}\right) \Phi_{0}\right\rangle & =0, \quad \text { for }\left\langle X^{\perp}, X\right\rangle=0, \\
\exp (2 \pi d(X)) \Phi_{0} & =\Phi_{0},
\end{aligned}
$$

where the derivative of $V[\Phi]$ is defined in Eq. (7).

Consider a vortex solution, defined by $\left(\Phi_{0}, X\right)$, satisfying the above three conditions. Two specific gauge choices have been made: firstly, it is in the temporal gauge; and, secondly, the gauge is such that the solution is axially symmetric. However, there is still residual gauge freedom defined from the global gauge transformations of $G$.

To track the gauge equivalence of solutions we shall compare solutions that also share the same $\Phi_{0}$. Then the relevant group is the subgroup of global gauge transformations defined by $G$ that leave $\Phi_{0}$ invariant. Clearly this is defined by $H$.

Thus the gauge freedom of solution (13a), (13b) is given by the group $H$, with transformation:

$$
\Phi(r, \theta) \mapsto D(h) \Phi(r, \theta)=f_{\mathrm{NO}}(X ; r) D[\exp (\operatorname{Ad}(h) X \theta)] \Phi_{0},
$$

$$
\underline{A}(r, \theta) \mapsto \operatorname{Ad}(h) \underline{A}=\frac{g_{\mathrm{NO}}(X ; r)}{r} \operatorname{Ad}(h) X \underline{\hat{\theta}} .
$$

This is equivalent to transforming the pair:

$$
\left(X, \Phi_{0}\right) \mapsto\left(\operatorname{Ad}(h) X, \Phi_{0}\right) .
$$

Hence, all the pairs $\left\{\left(\operatorname{Ad}(h) X, \Phi_{0}\right): h \in H\right\}$ define vortex solutions that are gauge equivalent. If two vortices may not be so related then they are gauge inequivalent.

The origin of family structure is now transparent. In general we write

$$
\mathcal{G}=\mathcal{H} \oplus \mathcal{M},
$$

with the orthogonality defined by Eq. (4). This is the reductive decomposition and has the following properties:

$$
[\mathcal{H}, \mathcal{H}] \subseteq \mathcal{H} \quad \text { and } \quad[\mathcal{H}, \mathcal{M}] \subseteq \mathcal{M} .
$$

One interprets $\mathcal{M}$ as the space of generators defining massive gauge bosons. If $\mathcal{M}$ is reducible under $\operatorname{Ad}(H)$, it may be decomposed into orthogonal irreducible subspaces

$$
\mathcal{M}=\mathcal{M}_{1} \oplus \cdots \oplus \mathcal{M}_{N} .
$$

This is the origin of family structure: vortices with generators lying in the same $\mathcal{M}_{i}$ may be gauge equivalent; while two vortices with generators lying within different $\mathcal{M}_{i}$ 's may not be.

However, more importantly, the decomposition (19) allows one to classify all embedded vortex solutions:

(i) embedded vortices are defined by ${ }^{1} X \in \mathcal{M}_{i}$;

(ii) there may be additional combination vortex solutions lying within $\mathcal{M}_{i} \oplus \mathcal{M}_{j}$ for certain critical values of the coupling constants such that

$$
\frac{\left\|d\left(X_{1}\right) \Phi_{0}\right\|}{\left\|X_{i}\right\|}=\frac{\left\|d\left(X_{j}\right) \Phi_{0}\right\|}{\left\|X_{j}\right\|}
$$

with $X_{i} \in \mathcal{M}_{i}, X_{j} \in \mathcal{M}_{j}$.

We shall discuss the implications of these results before discussing the method of proof.

\section{A. Implications}

Consider a generator $X_{i} \in \mathcal{M}_{i}$ normalized such that $D\left(\exp \left(2 \pi X_{i}\right)\right) \Phi_{0}=\Phi_{0}$. Furthermore let this generator be minimal, so that $D\left(\exp \left(\theta X_{i}\right)\right) \Phi_{0} \neq \Phi_{0}$ for $\theta \in(0,2 \pi)$. The elements of this set all generate vortices of unit winding number, and define the manifold

$$
\operatorname{Ad}(H) X_{i} \subset \mathcal{M}_{i} .
$$

When $\operatorname{rank}\left(\mathcal{M}_{i}\right)=1, \operatorname{Ad}(H)$ is transitive over $\mathcal{M}_{i}$ and this manifold is a $k$ sphere with $k=\operatorname{dim}\left(\mathcal{M}_{i}\right)-1$.

\footnotetext{
${ }^{1}$ The situation is slightly more complicated for those $\mathcal{M}_{i}$ 's of nontrivial rank, as we discussed in the prenote.
} 
Similarly the corresponding vortex of winding number $n$ is generated by $n X_{i}$. Thus when combination vortices are not admitted as solutions, the total space of vortex generators is

$$
\left\{\sum_{n_{1} \neq 0} n_{1} \operatorname{Ad}(H) X_{1}\right\} \oplus \cdots \oplus\left\{\sum_{n_{N} \neq 0} n_{\mathrm{N}} \operatorname{Ad}(H) X_{N}\right\} .
$$

This represents a collection of concentric maximal spheres within each vector space $\mathcal{M}_{i}$, with the radii of the spheres being proportional to the winding numbers of vortices defined from generators within that sphere. Note that if any $\operatorname{rank}\left(\mathcal{M}_{i}\right)>1$ then the situation becomes more complex.

This picture is useful when considering gauge equivalence of vortices: gauge transformations of the form (15a), (15b) move vortex generators $X$ around the particular maximal sphere in which $X$ lies, taking $X \mapsto \operatorname{Ad}(h) X$. Such transformations have two distinct types: (i) when $\operatorname{dim} \mathcal{M}_{i}=1$ the space of vortex generators corresponds to a set of equally spaced points. The corresponding vortices are invariant under $H$; (ii) when $\operatorname{dim} \mathcal{M}_{i}>1$ the space of vortex generators corresponds to a set of concentric maximal spheres. The corresponding vortices are not invariant under $H$, with two vortices within the same sphere having generators related by $\operatorname{Ad}(H)$. Note that such vortices are gauge equivalent to their antivortex.

\section{B. Method of proof}

For pedagogical reasons we shall delay a proper treatment of the formalism and proof to another paper [3], and present here instead a sketch of how the proof proceeds.

We shall repeat again the conditions upon a generator $X$ of an embedded vortex of the form (13a), (13b): (i) the boundary conditions of the vortex form a closed geodesic on the vacuum manifold, so that with appropriate normalization $\exp (2 \pi X) \in H$; (ii) in order that the embedded vortex is a solution to the equations of motion one needs $\left\langle d(X) \Phi_{0}, d\left(X^{\perp}\right) \Phi_{0}\right\rangle=0$ for all $X^{\perp}$ such that $\left\langle X^{\perp}, X\right\rangle=0$.

Only vortex generators that satisfy these two conditions define embedded vortices. We shall firstly indicate how vortex generators in $\mathcal{M}_{i}$ are classified, before discussing combination vortices.

Vortex generators that satisfy (i) above are classified by the following conjecture:

Conjecture 1. For $X \in \mathcal{M}_{i}$, the geodesic $\gamma_{X}(\theta)$ $=\left\{D\left(e^{X \theta}\right) \Phi_{0}: \theta \in \mathbf{R}\right\}$ defined from $X$ is closed. Note that we are restricting $\mathcal{M}_{i}$ such that $\operatorname{rank}\left(\mathcal{M}_{i}\right)=1$.

Proof of this result is discussed in [3] for a wide variety of cases by relating it to the embeddings of maximal Abelian subalgebras within $\mathcal{G}$. These maximal Abelian subalgebras generate toroidal submanifolds of the vacuum manifold, and the closed geodesics within such toroidal submanifolds are easily found.

Vortex generators satisfying condition (ii) are categorized by the following theorem:

Theorem 2. The (real) inner product on $\mathcal{V}$ is related to the inner product on $\mathcal{M}$ by

$$
\begin{gathered}
\left\langle d\left(X_{i}\right) \Phi_{0}, d\left(Y_{j}\right) \Phi_{0}\right\rangle=\lambda_{i} \lambda_{j}\left(X_{i}, Y_{j}\right), \quad X_{i} \in \mathcal{M}_{i}, Y_{j} \in \mathcal{M}_{j}, \\
\text { where } \lambda_{i}=\frac{\left\|d\left(X_{i}\right) \Phi_{0}\right\|}{\left\|X_{i}\right\|} .
\end{gathered}
$$

Each $\lambda_{i}$ is constant upon its particular $\mathcal{M}_{i}$.

This theorem is proved in [3] by relating it to the conformal properties of inner products.

We now consider combination vortices. These are vortices with generators lying between, say, $\mathcal{M}_{i}$ and $\mathcal{M}_{j}$. Generally the generator of a combination vortex may be written $X=\alpha X_{i}+\beta X_{j}$, where $X_{i} \in \mathcal{M}_{i}$ and $X_{j} \in \mathcal{M}_{j}$. The spectrum of these that close is generally rather complicated, and may be either a continuous spectrum between $\mathcal{M}_{i}$ and $\mathcal{M}_{j}$ or a discrete one.

For $\left\langle d(X) \Phi_{0}, d\left(X^{\perp}\right) \Phi_{0}\right\rangle=0$ to be satisfied for combination vortices one need only consider $X^{\perp}=X_{i} /\left(\alpha\left\|X_{i}\right\|^{2}\right)$ $-X_{j} /\left(\beta\left\|X_{j}\right\|^{2}\right)$; then direct substitution yields the following condition:

$$
\frac{\left\|d\left(X_{i}\right) \Phi_{0}\right\|}{\left\|X_{i}\right\|}=\frac{\left\|d\left(X_{j}\right) \Phi_{0}\right\|}{\left\|X_{j}\right\|}
$$

This simple condition deserves some comment. The expression $\left\|d(X) \Phi_{0}\right\| /\|X\|$ is independent of the generator $X \in \mathcal{M}_{i}$ (by scaling and adjoint action), and is thus dependent only upon the coupling constants acting as scales in the inner product (4). One thus interprets condition (23) as a restriction upon the ratios of gauge coupling constants that are allowed in order to admit embedded combination vortex solutions.

\section{STABILITY OF EMBEDDED VORTICES}

After determining the family structure, one wishes to determine how this spectrum of vortices relates to the stability of embedded defects. There are two types of stability to consider: (i) topological stability, quantified by the first homotopy group of the vacuum manifold. Such vortices may either be Abelian or non-Abelian as specified below; (ii) dynamical stability [4], which occurs when the theory admits stable semilocal vortices in a limit (the semilocal limit) of the coupling constants. By continuity dynamical stability occurs for a finite region of coupling constant space around the region of stable semilocal vortices.

Firstly, we shall remind the reader of some concepts that will prove useful to the following discussion. Recall that the center $\mathcal{C}$ of $\mathcal{G}$ is the set of elements that commute with $\mathcal{G}$. Then the stability of vortices is related to the projection of $\mathcal{C}$ onto $\mathcal{M}$,

$$
\operatorname{pr}_{\mathcal{M}}(X)=X+X_{h},
$$

with $X_{h} \in \mathcal{H}$ the unique element such that $\operatorname{pr}_{\mathcal{M}}(X) \in \mathcal{M}$. We shall prove later that $\operatorname{pr}_{\mathcal{M}}(\mathcal{C})$ consists of one-dimensional irreducible $\mathcal{M}_{i}$ 's.

The main result of this section is that $\operatorname{pr}_{\mathcal{M}}(\mathcal{C})$ differentiates between Abelian topologically stable or dynamically stable embedded vortices. The distinction being (1) topologically stable Abelian vortices are generated by elements in the 
intersection of $\mathcal{M}$ and $\mathcal{C}$ and (2) dynamically stable vortices (in some coupling constant limit) are generated elements in the nontrivial projection of $\mathcal{C}$ onto $\mathcal{M}$. Such vortices always correspond to one-dimensional $\mathcal{M}_{i}$ 's.

\section{A. Topologically stable Abelian vortices}

Abelian topological vortices are present for a symmetry breaking of the form

$$
G=G^{\prime} \times U(1)_{1} \times \cdots \times U(1)_{N} \rightarrow H \subseteq G^{\prime} .
$$

So that the symmetry breaking is the product of $U(1)_{1}$ $\times \cdots \times U(1)_{N} \rightarrow 1$ and $G^{\prime} \rightarrow H$, with there being no Abelian part of $G^{\prime}$ with the subalgebra $u(1) \subseteq \mathcal{M}$. The first half of the symmetry breaking gives a nontrivial topology, with the topological charges being elements of $\mathbf{Z}^{N}$.

The Lie algebra of group $G$ decomposes under the adjoint action of $H$ as

$$
\mathcal{G}=\mathcal{H} \oplus \mathcal{M}^{\prime} \oplus \mathcal{M}_{1} \oplus \cdots \oplus \mathcal{M}_{N},
$$

where

$$
\mathcal{M}_{i}=u(1)_{i} .
$$

The individual spaces $\mathcal{M}_{1}, \ldots, \mathcal{M}_{N}$ are clearly irreducible under $\operatorname{Ad}(H)$ and are thus one-dimensional irreducible parts of $\mathcal{M}$. In addition, $\mathcal{M}^{\prime}$ may decompose further under $\operatorname{Ad}(H)$; however, this is irrelevant for the present discussion.

Therefore the generators of stable Abelian vortices $\mathcal{M}_{1}$ $\oplus \cdots \oplus \mathcal{M}_{N}$ lie within the intersection of the Lie algebra of the center of $G$ with $\mathcal{M}$, i.e.,

$$
\mathcal{M}_{1} \oplus \cdots \oplus \mathcal{M}_{N}=\mathcal{M} \cap \mathcal{C} .
$$

\section{B. Topological stability from $S / Z_{n}$}

Topological stability can arise from elements outside the center of the group if discrete symmetries are involved. For example when $G \rightarrow H$ such that

$$
\frac{G}{H} \cong \frac{S}{Z_{n}} \times \cdots
$$

with $S$ a simply connected manifold of dimension larger than one, then one has non-Abelian topologically stable vortex solutions. The topological charges are given by the equivalence classes of the first homotopy group

$$
\pi_{1}\left(\frac{\mathcal{M}}{\mathbf{Z}_{n}}\right)=\mathbf{Z}_{n}
$$

However, for $S$ not simply connected one may have a more complex structure, see for instance ${ }^{3} \mathrm{He}$ in [2].

\section{Dynamical stability}

A vortex may be dynamically stable [4] if there exists a limit of the coupling constants $\left\{q_{i}\right\}$ such that the model then admits stable semilocal vortices-i.e., in that limit it has a breaking of the form below in Eq. (31). We refer to such a limit as the semilocal limit; in that limit some of the coupling constants vanish, so that the corresponding symmetry becomes global. By continuity, vortices are dynamically stable for some finite region in coupling constant space around the semilocal limit.

Preskill [5] has shown how to construct semilocal defects generally. For vortices, one needs a symmetry breaking scheme of the form

$$
G_{\text {global }} \times U(1)_{\text {local }} \rightarrow H, \quad \text { with } H \cap U(1)_{\text {local }}=1,
$$

where the suffixes "global" and "local" represent nongauge and gauged symmetries, respectively. Requiring that this does not admit topological vortices leads to the condition that $H \nsubseteq G_{\text {global }}$. Note that Eq. (31) needs only be a subpart of a more general symmetry breaking.

The main result of this section is motivated through observing that we are trying to isolate part of the symmetry breaking and then take a limit in which this part of the symmetry breaking resembles Eq. (31). That $H \nsubseteq G_{\text {global }}$ implies that one of the generators of $\mathcal{H}$ is a linear combination of generators in $\mathcal{C}$ and $\mathcal{G}_{\text {global }}$. The other linear combination, which is perpendicular to this, lies in $\mathcal{M}$ and is the clear contender for vortex generators generating dynamically stable embedded vortices.

The result is the following.

Theorem 3. Vortex generators $X \in \operatorname{pr}_{\mathcal{M}}(\mathcal{C})$ such that $X \notin \mathcal{C}$ define embedded vortices that are stable in a well-defined semi-local limit of the model and are thus dynamically stable for a region of parameter space around that semilocal limit.

Proof. Consider $X \in \operatorname{pr}_{\mathcal{M}}(\mathcal{C})$, such that it generates a closed geodesic. Then $X=X_{c}+X_{h}$, with $X_{c} \in \mathcal{C}$, such that $\operatorname{pr}_{\mathcal{M}}\left(X_{c}\right)=X$, generating a $U(1)_{c} \subseteq C$ and $X_{h} \in \mathcal{H}$ generating a $U(1)_{h} \subset H$. These define a decomposition of the symmetry breaking of the Lie algebras:

$$
\mathcal{G}=\mathcal{G}^{\prime} \oplus u(1)_{c} \rightarrow \mathcal{H}=\mathcal{H}^{\prime} \oplus u(1)_{h},
$$

with $U(1)_{c} \cap H=1$. It is now clear that the appropriate semilocal limit to obtain a symmetry breaking of the form Eq. (31) is to make coupling constants appertaining to $G^{\prime}$ vanish. The corresponding vortex generator is $X$. This completes the proof.

We also have a useful subresult about the dimension of $\mathcal{M}_{i}$ 's for such vortices:

Lemma. Vortices with a stable semilocal limit are always generated by generators in one dimensional $\mathcal{M}_{i}$ 's.

Proof. The proof that if $\mathcal{M}_{i} \subseteq \operatorname{pr}_{\mathcal{M}}(\mathcal{C})$ then $\operatorname{dim}\left(\mathcal{M}_{i}\right)=1$ relies on the following identification: if one writes $\mathcal{M}_{\mathrm{ker}}$ as the collection of one dimensional $\mathcal{M}_{i}$ 's then necessarily $\mathcal{M}_{\text {ker }}=\{X \in \mathcal{M}: \operatorname{Ad}(H) X=X\}$. Now we are reduced to showing $\operatorname{pr}_{\mathcal{M}}(\mathcal{C}) \subseteq \mathcal{M}_{\mathrm{ker}}$.

Consider $\left(X_{c}+X_{h}\right) \in \operatorname{pr}_{\mathcal{M}}(\mathcal{C})$, with $X_{c} \in \mathcal{C}$ and $X_{h} \in \mathcal{H}$. By Eq. (18), $\left[\mathcal{H}, X_{c}+X_{h}\right] \in \mathcal{M}$. But also $\left[\mathcal{H}, X_{c}+X_{h}\right]=\left[\mathcal{H}, X_{h}\right]$ $\in \mathcal{H}$. Hence $\left[\mathcal{H}, X_{c}+X_{h}\right]=0$, and equivalently $\operatorname{Ad}(H)\left(X_{c}\right.$ $\left.+X_{h}\right)=X_{c}+X_{h}$, proving the result. 


\section{EXTENSIONS TO DOMAIN WALLS AND MONOPOLES}

So far we have considered only how the properties of embedded vortices are related to the underlying group theory of the formalism introduced in [1]. However, that formalism is also relevant to embedded domain walls and embedded monopoles. Hence, we discuss in this section how our approach may be extended to cover them. One should note that a considerably expanded version of this discussion will be covered in [6].

Nontopological embedded domain walls and embedded monopoles are unstable. Nontopological embedded monopoles are unstable due to a long range instability of the magnetic field $[7,8]$; which is related to the nonexistence of semilocal monopoles [5]. Nontopological embedded domain walls are unstable due to a short range instability of the scalar field [1]. This contrasts strongly with the case of nontopological embedded vortices, where one may still have dynamical stability $[4,5]$.

Thus we shall only discuss the nature of the family stability for embedded domain walls and monopoles.

We deal with embedded domain walls and embedded monopoles separately.

\section{A. Embedded domain walls}

An embedded domain wall solution is defined from the singleton $\Phi_{0} \in \mathcal{V}$ :

$$
\begin{gathered}
\Phi(x)=\tanh (c x) \Phi_{0}, \\
A^{\mu}=0,
\end{gathered}
$$

where $c$ is some constant depending upon parameters in the Lagrangian. This is related to the embedded subtheory formalism by

$$
\begin{aligned}
& G_{\mathrm{emb}}=\mathbf{Z}_{2}, \\
& H_{\mathrm{emb}}=\mathbf{1}, \\
& \mathcal{V}_{\mathrm{emb}}=\mathbf{R} \Phi_{0} .
\end{aligned}
$$

Hence, provided that $G$ is transitive over the vacuum manifold all embedded domain walls are gauge equivalent.

\section{B. Embedded monopoles}

An embedded monopole solution is defined from the embedding

$$
\begin{gathered}
G \rightarrow H \\
\cup \cup \\
S U(2) \rightarrow U(1) .
\end{gathered}
$$

With the embedded solution specified by the triplet $\left(\Phi_{0}, X_{1}, X_{2}\right) \in \mathcal{V} \times \mathcal{M} \times \mathcal{M}$, with the following constraints on the pair $\left(X_{1}, X_{2}\right) \in \mathcal{M} \times \mathcal{M}$ :

(i) the pair $\left(X_{1}, X_{2}\right)$ consists of two members of an orthogonal basis of an $s u(2) \subset \mathcal{G}$, thus

$$
\left\|X_{1}\right\|=\left\|X_{2}\right\|, \quad\left\langle X_{1}, X_{2}\right\rangle=0,
$$

and

$$
\left[X_{1},\left[X_{1}, X_{2}\right]\right] \propto X_{2}, \quad\left[X_{2},\left[X_{1}, X_{2}\right]\right] \propto X_{1} .
$$

(ii) the embedded $S U(2)$ is such that $S U(2) \cap H=U(1)$, thus

$$
\left[X_{1}, X_{2}\right] \in \mathcal{H}
$$

(iii) the generators are properly normalized so that, for $i$ $=\{1,2\}$,

$$
\exp \left(2 \pi X_{i}\right) \Phi_{0}=\Phi_{0}
$$

Then the corresponding monopole solution (for winding number $n=1$ ) is a usual $S U(2)$ hedgehog configuration embedded in $G \rightarrow H$

$$
\begin{aligned}
\underline{\Phi}(\underline{r}) & =f_{\text {mon }}(r) \underline{\hat{r}}, \\
A_{a}^{\mu}(\underline{r}) & =\frac{g_{\text {mon }}(r)}{r} \epsilon_{\mu a b} X_{b},
\end{aligned}
$$

where $f_{\text {mon }}(r)$ and $g_{\text {mon }}(r)$ are the usual monopole profile functions. Notationally, we are treating $\Phi$ to be a vector within its corresponding embedded subtheory and we are using $X_{3}=\left[X_{1}, X_{2}\right]$.

The corresponding embedded subtheory is defined by the triplet $\left(\Phi_{0}, X_{1}, X_{2}\right)$ :

$$
\begin{aligned}
& G_{\mathrm{emb}}=\exp \left(\mathbf{R} X_{1} \oplus \mathbf{R} X_{2} \oplus \mathbf{R}\left[X_{1}, X_{2}\right]\right), \\
& H_{\mathrm{emb}}=\exp \left(\mathbf{R}\left[X_{1}, X_{2}\right]\right), \\
& \mathcal{V}_{\mathrm{emb}}=\mathbf{R} D\left(G_{\mathrm{emb}}\right) \Phi_{0} .
\end{aligned}
$$

We now discuss the family structure of embedded monopoles by relating them to the family structure of embedded vortices with the following observation:

$$
\begin{gathered}
G \rightarrow H \\
\cup \quad \cup \\
S U(2) \rightarrow U(1) \\
\cup \quad \cup \\
U(1) \rightarrow \mathbf{1 .}
\end{gathered}
$$

Thus monopole solutions always contain embedded vortex solutions. Using the results on embedded vortices we may immediately infer

$$
\left(X_{1}, X_{2}\right) \in \mathcal{M}_{i} \times \mathcal{M}_{i}
$$

Therefore monopoles defined from different $\mathcal{M}_{i}$ 's are gauge inequivalent. There could also be further family structure arising from gauge inequivalent monopole configurations with different pairs $\left(X_{1}, X_{2}\right)$ within the same irreducible subspace $\mathcal{M}_{i}$.

The structure of embedded monopole solutions is discussed more fully in a forthcoming manuscript [6]. 


\section{CONCLUSIONS}

We conclude by summarizing our main results.

(1) In Sec. II we expanded on the embedded defect formalism of [1] by making the underlying group theory explicit.

(2) In Sec. III we showed that the family structure of embedded vortices is dependent only upon the group theory of symmetry breaking. We derived how so, in a prescriptive way that allows a determination of the family structure.

(3) In Sec. IV we related this family structure to stability. Classifying stability into (Abelian and non-Abelian) topological stability and dynamical stability, we showed how Abelian topological stability comes from the intersection of the center of the gauge group with vortex generators. Correspondingly dynamical stability originates from the nontrivial projection of the center.

(4) In Sec. V we showed how points (2) and (3) above can be carried over to embedded domain walls and embedded monopoles.

\section{ACKNOWLEDGMENTS}

This work was supported partly by PPARC and partly by the European Commission under the Human Capital and Mobility program. One of us (N.F.L.) acknowledges EPSRC for financial support. We wish to thank N. Manton and T. Vachaspati for interesting discussions related to this work when it was in a preliminary state of development, we would also like to thank T. Kibble and N. Turok for interesting discussions and important clarification when this work was in an advanced state.

\section{APPENDIX: ON THE NIELSEN-OLESEN PROFILE FUNCTIONS}

The Nielsen-Olesen Functions are the (scaled) profiles of the scalar and gauge fields within the vortex solution. They depend upon the scalar quartic self-coupling and the charge, through the parameters in the Lagrangian describing the field theory. Although we have tried to avoid referring to the actual underlying field theory in this paper, it becomes necessary when discussing the profile functions.

The Nielsen-Olesen profile functions are defined from the vortex solutions in the Abelian-Higgs model. The AbelianHiggs model is defined through the Lagrangian, which describes the interaction between the Higgs field $\Phi \in \mathbf{C}$ and the gauge field $A_{\mu}$ :

$$
\begin{aligned}
\mathcal{L}\left[\lambda, q ; \Phi, A_{\mu}\right] & \\
& =\frac{-1}{4} F_{\mu \nu}^{*} F^{\mu \nu}+\frac{1}{2} D_{\mu} \Phi^{*} D^{\mu} \Phi-\lambda\left(\Phi * \Phi-\eta^{2}\right)^{2},
\end{aligned}
$$

where

$$
\begin{gathered}
D_{\mu}=\partial_{\mu}+q A_{\mu}, \\
F_{\mu \nu}=\partial_{\nu} A_{\mu}-\partial_{\mu} A_{\nu} .
\end{gathered}
$$

Substitution of the ansatz for the vortex (of winding number n)

$$
\begin{aligned}
& \Phi(r, \theta)=\eta f(\lambda, q, n ; r) e^{i n \theta}, \\
& \underline{A}(r, \theta)=g(\lambda, q, n ; r)\left(\frac{i n}{q r}\right) \underline{\hat{\theta}}, \quad A_{0}=0,
\end{aligned}
$$

yields the Lagrangian for $f, g$ :

$$
\begin{aligned}
\mathcal{L}[\lambda, q ; f, g]= & -\frac{n^{2}}{4 q^{2}} \frac{1}{r^{2}}\left(\frac{d q}{d r}\right)^{2}-\frac{\eta^{2}}{2}\left(\frac{d f}{d r}\right)^{2} \\
& -\frac{n^{2} \eta^{2} f^{2}}{2 r^{2}}(1+g)^{2}-\lambda \eta^{4}\left(f^{2}-1\right)^{2} .
\end{aligned}
$$

The Nielsen-Olesen profile functions $f_{\mathrm{NO}}$ and $g_{\mathrm{NO}}$ minimize this Lagrangian.

A useful identity that relates the profile functions at different values of the electric charge is

$$
\begin{aligned}
& f_{\mathrm{NO}}\left(\lambda, q^{\prime}, n ; r\right)=f_{\mathrm{NO}}\left(\frac{\lambda}{\left(q^{\prime} / q\right)^{2}}, q, n ; \frac{q^{\prime}}{q} r\right), \\
& g_{\mathrm{NO}}\left(\lambda, q^{\prime}, n ; r\right)=g_{\mathrm{NO}}\left(\frac{\lambda}{\left(q^{\prime} / q\right)^{2}}, q, n ; \frac{q^{\prime}}{q} r\right) .
\end{aligned}
$$

Proof is provided by direct substitution into the Lagrangian.

In certain situations the profile functions for a general embedded vortex may be related back to the Nielsen-Olesen profile functions for the Abelian-Higgs model. Then one has recourse to the above identity (A5a), (A5b) to relate the profile functions between different classes.

To examine the form of a general embedded vortex one needs to use the Lagrangian for a general Yang-Mills theory. The Lagrangian is of the form

$$
\mathcal{L}=\frac{-1}{4}\left\langle F_{\mu \nu}, F^{\mu \nu}\right\rangle+\frac{1}{2}\left\langle D_{\mu} \Phi, D^{\mu} \Phi\right\rangle-V(\Phi),
$$

where

$$
\begin{aligned}
D_{\mu} & =\partial_{\mu}+d\left(A_{\mu}\right), \\
F_{\mu \nu} & =\partial_{\nu} A_{\mu}-\partial_{\mu} A_{\nu}-\left[A_{\mu}, A_{\nu}\right] .
\end{aligned}
$$

Here $\langle.,$.$\rangle are the respective inner products and V(\Phi)$ is the scalar potential, which is quartic in the components of $\Phi$. Note that we are using the scaled representation, which naturally introduces a coupling into the non-Abelian term of $F^{\mu \nu}$ by constraining $d\left(\left[A^{\mu}, A^{\nu}\right]\right)=\left[d\left(A^{\mu}\right), d\left(A^{\nu}\right)\right]$.

Consider the ansatz for an embedded defect solution

$$
\Phi(r, \theta)=f(X ; r) e^{d(X) \theta} \Phi_{0},
$$




$$
\underline{A}(r, \theta)=\frac{g(X ; r)}{r} X \underline{\hat{\theta}}, \quad A_{0}=0 .
$$

Since $V(\Phi)$ is a scalar potential, and we implicitly assume that the asymptotics of the embedded defect lie in the physical vacuum manifold, which is the minimum of the potential, we must have

$$
V(\Phi(r, \theta))=\lambda\left[\Phi_{0}\right] \eta^{4}\left(f(r ; X)^{2}-1\right)^{2},
$$

with $\eta=\left\|\Phi_{0}\right\|$. We shall assume the vacuum manifold is connected, then $\lambda$ is independent of $\Phi_{0}$ and $\eta$ is independent of the direction of $\Phi_{0}$.

Elementary substitution of the ansatz into the Lagrangian yields the Lagrangian describing the profile functions $f, g$ :

$$
\begin{aligned}
\mathcal{L}[\lambda, X ; f(X ; r), g(X ; r)] & \\
= & -\frac{\|X\|^{2}}{4 r^{2}}\left(\frac{d g}{d r}\right)^{2}-\frac{\eta^{2}}{2}\left(\frac{d f}{d r}\right)^{2} \\
& -\left\|d(X) \Phi_{0}\right\|^{2} \frac{f^{2}}{2 r^{2}}(1+g)^{2}-\lambda \eta^{4}\left(f^{2}-1\right)^{2},
\end{aligned}
$$

and as before the Lagrangian is minimized by the NielsenOlesen profile functions $f_{\mathrm{NO}}$ and $g_{\mathrm{NO}}$.

Now, providing

$$
\frac{\left\|d(X) \Phi_{0}\right\|}{\left\|\Phi_{0}\right\|}=n, \quad \text { with } n \in Z
$$

the winding number of the defect, then we have a simple relation between profile functions of embedded defects and the profile functions in the Abelian-Higgs model. One corresponds the embedded defects profile functions to those of the Abelian-Higgs model with charge and winding number:

$$
q^{2}=\frac{\left\|d(X) \Phi_{0}\right\|^{2}}{\left\|\Phi_{0}\right\|^{2}\|X\|^{2}}
$$

$$
n=\frac{\left\|d(X) \Phi_{0}\right\|}{\left\|\Phi_{0}\right\|} .
$$

We also have use of the identity (A5a), (A5b) for relating embedded defects in different classes. Providing the condition (A11) is satisfied, and the winding number of two embedded vortices in different classes are equal (the two vortices being defined by $X_{1}$ and $X_{2}$ ) then

$$
\begin{aligned}
f_{\mathrm{NO}}\left(\lambda, X_{1} ; r\right) & =f_{\mathrm{NO}}\left(\frac{\lambda}{\gamma^{2}}, X_{2} ; \gamma r\right), \\
g_{\mathrm{NO}}\left(\lambda, X_{1} ; r\right) & =g_{\mathrm{NO}}\left(\frac{\lambda}{\gamma^{2}}, X_{2} ; \gamma r\right), \\
\text { where } \gamma & =\frac{\left\|d\left(X_{1}\right) \Phi_{0}\right\|\left\|X_{2}\right\|}{\left\|d\left(X_{2}\right) \Phi_{0}\right\|\left\|X_{1}\right\|} .
\end{aligned}
$$

In the case of combination vortices [see Eq. (23)] the factor $\gamma$ is one (by definition) and the profile functions naturally coincide. This is to be expected since in many cases the vortices in different classes may be continuously deformed into one another when combination vortices exist.

It would be useful to know when the above condition (A11) is generally satisfied. The condition does not seem to be true generally, and one may construct realistic counter examples. However, there are also many theories which act as examples, for example the Weinberg-Salam theory.

It should be noted that condition (A11) is equivalent to the following condition:

$$
\begin{array}{r}
e^{2 \pi d(X)} \Phi_{0}=\Phi_{0}, \quad \text { and } X \in \mathcal{M}_{i}, \\
\text { and } \frac{\left\|d(X) \Phi_{0}\right\|}{\left\|\Phi_{0}\right\|} \in \mathbf{Z},
\end{array}
$$

i.e., when do the generators act like real numbers upon exponentiation? As a simple example the Pauli spin matrices do.
[1] M. Barriola, T. Vachaspati, and M. Bucher, Phys. Rev. D 50, 2819 (1994).

[2] N. F. Lepora and A. C. Davis, following paper, Phys. Rev. D 58, 125028 (1998).

[3] T. Kibble and N. F. Lepora (in preparation).

[4] T. Vachaspati and Ana Achúcarro, Phys. Rev. D 44, 3067
(1991); M. Hindmarsh, Nucl. Phys. B392, 461 (1993).

[5] J. Preskill, Phys. Rev. D 46, 4218 (1992).

[6] N. F. Lepora (in preparation).

[7] R. Brandt and F. Neri, Nucl. Phys. B161, 253 (1979).

[8] S. Coleman, The Unity of the Fundamental Interactions (Plenum, New York, 1983). 